\title{
Inspection of Existing Bridges - Moving on from condition rating
}

\author{
Rade Hajdin \\ Infrastructure Management Consultants $\mathrm{GmbH}$, Zürich, Switzerland \\ Faculty of Civil Engineering, University of Belgrade, Serbia
}

\author{
Joan Ramon Casas \\ UPC-BarcelonaTech, Civil and Environmental Engineering, Barcelona, Spain
}

\author{
Jose Matos \\ Universidade do Minho - Campus de Azurem, Guimarães, Portugal
}

Contacting author: Rade.Hajdin@imc-ch.com

\begin{abstract}
It is widely accepted that safety and serviceability are primary concerns in bridge design. However, for the most of bridges' service life, these concerns are addressed indirectly by a qualitative measure, defined herein as condition rating, which is based upon observable damages recorded during inspections. Condition rating is at best, only loosely correlated to safety and serviceability. It would be more reasonable to address safety and serviceability in an inspection process directly, using the information on bridge performance obtained during the design and construction.

To address this issue, the reliability was chosen as a Key Performance Indicators (KPI) for existing bridges and a novel practical solution is proposed. It is based on survey of observation types (visible defects, measurements, etc.) used in Europe, which were examined with regard to their potential impact on reliability regarding safety and serviceability. The impact of these observations on reliability is also dependent on their type, location and intensity/extent as well as on bridge structural systems. The paper presents a methodology to assess reliability, which heavily relies on data from design and construction phase. It also proposes a set of data elaborated in design and/or construction phase that need to enter current bridge data bases to allow a rough reliability assessment of existing bridges.
\end{abstract}

Keywords: condition, decision making, KPI, inspection, reliability, existing bridges, Bayesian net

\section{Introduction}

There is a broad consensus that the benefits of road infrastructure for the society cannot be overestimated. The investments in road infrastructure raise the growth potential of a national economy, which is realized by efficient exploitation of the road infrastructure. The road infrastructure enables road users to be involved in various productive activities that yield private, public and social goods. Maintaining these benefits on the long run in economically efficient, environmentally responsible 
and socially reconcilable manner is the fundamental task of road authorities. Bridges are critical components of the road infrastructure as they ensure fast safe passages over otherwise hardly surmountable obstacles. From the users' perspective, it is irrelevant whether a road is carried by the bridge or being in tunnel or merely resting on the soil, so long it provides the safe and fast travel from origin to destination. In this context, it is necessary to define what is meant by fast and safe.

There are codes of practice that apply to the design of a road infrastructure and they are related to clearance, speed, and weight allowance. The design travel speed defines the minimum legal travel time on an arbitrary road link. In reality, this minimum travel time can be achieved only in the case of unrestricted traffic flow i.e. if road capacity is sufficiently higher than traffic volume. Based on the current or future traffic, one can specify some travel time - exceeding the minimum travel time as the "fast travel". Clearly, the actual travel times will scatter significantly, and the measure of this scatter needs to be considered when defining "fast travel". In this context, one often refers to travel time reliability.

The safe travel however is somewhat difficult to define since it doesn't imply absolute protection from undesired events. When travelling, there is always a probability of undesired events, which can harm life and limb, induce economic losses and damage environment. The sufficiently low probability of such undesired events defines "safe travel".

The bridges can play significant role regarding the travel time since the failed or posted bridge can lead to detours and therefore to increased travel time. Furthermore, bridges or parts of them can fail/collapse harming life and limb and inducing adverse consequences for economy and environment. It is therefore not surprising that the structural safety is the primary concern of bridge owners since it affects both safe and fast travel. Besides structural safety the bridge owners care about serviceability, which relates to user comfort that can be affected by deflections and vibrations of a bridge. If safety or serviceability requirements are not met, the bridge will be posted or even closed with aforementioned consequences.
Following the definition of the "safe travel", a bridge is regarded as structurally safe if the probability of failure during its service life doesn't exceed some nominal value. Similar approach applies to serviceability in which the exceedance probability of some service limits (deflection, frequency, etc.) must be sufficiently low. Most modern codes of practice for bridge design have adopted this concept (e.g. [1]).

The owners need to ensure that their bridge inventory fulfils the safety and serviceability requirements during their service life and therefore maintain the benefits of road infrastructure. Considering the extent of the road infrastructure in developed countries, this is increasingly challenging task. The tools and methods currently available to owners to fulfil this task seem not to be adequate and in this paper some improvements are suggested.

\section{Current inspection practice}

Bridge Inspection practice differs quite significantly from country to country, but the common denominator is that it relies on visual inspections. The visual inspections are - if performed by a qualified structural engineer - cost efficient and very valuable source of information. During the inspection, the observation is recorded and evaluated. The result of inspections is qualitative indicator, which is named differently from country to country as condition rating, condition state, condition class, etc. Herein, the term condition rating is used. Whereas in a design phase the safety and serviceability concerns are addressed directly in quantitative manner, in a service phase, based on inspection results the condition rating is determined. The condition rating is a vague measure for the deviation of inspected bridge from the "as new" condition. The direct assessment of safety and serviceability is regarded as not cost efficient since it is commonly assumed that it always requires an in-depth material investigations and structural analysis.

Based on condition rating, owners trigger often costly in-depth investigations or even maintenance actions. In practice, once an in-depth investigation based on condition rating is triggered, the maintenance intervention is very likely to follow, even if a bridge can still be used without restrictions. The reasons are different from country to country, but 
one is surely the visual appearance and related perception of safety that entice owners to remove all visible damages with appropriate maintenance interventions. In some (rare) cases, a maintenance action is triggered if a bridge fails structural safety and serviceability checks, with the load and resistance models for the design of new bridges. This is clearly inadequate and uneconomical, given the remaining service life and possibilities to reduce uncertainties related to existing bridges. Thus, some countries have introduced safety and serviceability checking rules for existing bridges that differs from ones for the new bridges (e.g [2]).

The current approach seems not to be very logical. In the design phase, the wealth of information about safety and serviceability for different load situations is created. This information is unstructured and mostly in paper form. After the commissioning of newly constructed bridge, the documents containing this information is handed over to the bridges' owners or operators that act further on as trustees of the bridges assigned to them. The documents are mostly in archives and in general not easily accessible. During the service life, inspections are performed with no consideration of safety and serviceability information produced during the design phase. It is only within the in-depth investigation that the safety and serviceability are assessed again. There is a substantial gap during the life span of a bridge, in which decision are made based on qualitative indicators, that are sometimes unrelated to the key concerns of bridge owners: safety and serviceability.

In most countries, bridge management is supported by databases, in which the results of inspections are stored, sometimes in great detail. The information from design phase i.e. critical load combinations, safety factors, assumed traffic loads is usually not stored in these databases. In some road agencies, there are load rating software that facilitate evaluation of special transports and therefore include some information from design phase, but it is rarely used in conjunction with inspection results.

Even more surprising is that the relevant information on safety and serviceability is commonly not stored in the database after maintenance interventions. It can be assumed that the provisions of the current code of practice are fulfilled due to maintenance interventions, but it is not recorded if these are exceeded and by what margin.

It should be noted that within the in-depth investigations a substantial work effort is necessary to find information from the design phase or previous maintenance activities. In some cases, the information on existing bridges is lost due to negligence or some accident (e.g. fire, flooding).

In some ways, the current bridge management undergoes amnesia because

- relevant information from the design phase and/or in-depth investigation is not stored and/or

- information is stored only in paper form and is lost due to negligence or accidents.

It is not that bridge owners and operators are not aware of this deficiency, but to remedy it, they need to be provided tools and resources to efficiently store and access the elaborated information on their bridge inventory. In the time of growing awareness of data importance and big data, it is high time to establish an organizational setting for business processes to ensure the benefit of collected data on the long term.

\section{Improving benefit from inspections}

The challenging task of maintaining manifold benefits of road infrastructure, mentioned in chapter 1 can be only efficiently coped with, if the bridge owners have all necessary information at their fingertips. This means that the current databases need to be significantly improved to accommodate all relevant information from design, construction, inspections and maintenance interventions. This transition can be quite costly and therefore needs to be performed gradually.

\subsection{Safety and serviceability of undamaged bridges}

The information on safety and serviceability margin of an undamaged bridge is essential in the service phase. This information should be structured and include all relevant load cases, which would also allow owners to have a clear picture of possible failure modes and related vulnerable zones that need to be investigated in more detail. 
The current databases are not structured to accommodate the graphical representation of structural systems and load situations. The material properties and load effects need also to be included in the database as searchable data and coupled with graphical representations. The same apply to load models and provisions of current and previous codes of practice.

A large effort is required to obtain and store information for all existing bridges and this cannot be done within a short time period. Ideally, it could be done together with inspections or in-depth investigations and in this way, one can gradually fill the database. In particular, the database needs to support the evaluation of serviceability and safety margins against current design loads or loads beyond legal limits. These margins can be expressed therein either as

- the degree of compliance $n$, that can be evaluated for each load situation as a ratio between the available resistance/service limit and resistance/service requirements based on the total factored load effect, or as

- the traffic load capacity factor with which the traffic load can be multiplied and still fulfil the safety and serviceability requirements.

This information is very useful for the owners to qualitatively asses if a damage is relevant on a certain bridge and perhaps adapt the condition rating accordingly. However, given the large disparity between the uncertainties of degree of compliance and the interpretation of inspection findings, the quantitative assessment of the impact of inspection findings to the degree of compliance could be misleading. To consider this disparity adequately one needs to resort to probabilistic methods as proposed In COST Action TU 1406 [3].

\subsection{Reliability of undamaged bridges}

The modern design codes define the safety and serviceability in terms of reliability i.e. the probability that a bridge will be fit for purpose during its service life. In [1] the target annual reliability index $\beta$ for safety is 4.7 (corresponds to occurrence probability of $1.3 \cdot 10^{-6}$ ) and for serviceability 2.9 (corresponds to occurrence probability of $\left.1.9 \cdot 10^{-3}\right)$. The bridge is considered as safe and serviceable if specified reliability indexes are not below these target values.

The partial safety factors in [1] are calibrated for the target reliability indices, but this calibration is significantly influenced by the assumed characterization of uncertainty. Given that the uncertainties of actions defined in codes don't significantly vary, the partial safety factors in codes of practice will result in reliability indices that are not lower than the target values. Nevertheless, the evaluation of reliability may be economically beneficial if existing bridges can still be used without restrictions.

For the methodology described in this paper the evaluation of reliability of undamaged bridge due the current code loading is of particular interest as it serves as a baseline to assess the reliability of damaged bridge.

The reliability of an undamaged bridges modelled as simply supported beam can be for instance evaluated using the Bayesian network in Figure 1.

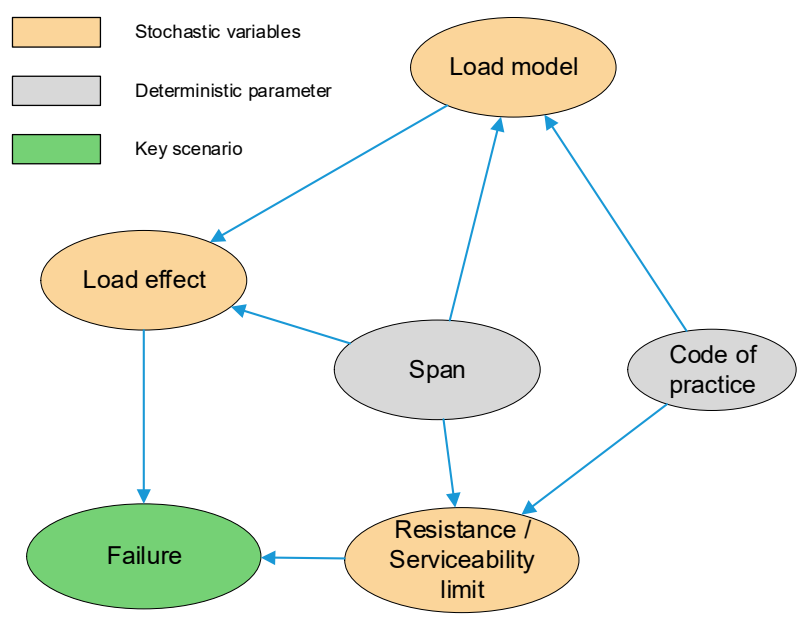

Figure 1. Reliability evaluation of undamaged bridge

To illustrate the presented approach, the safety of an existing bridge that is modelled as simply supported beam is assessed. It is assumed that the safety is given if the probability of failure doesn't exceed $1.3 \cdot 10^{-6}$.

The simply supported beam is presented in and the traffic load (node "Load model" in Figure 1) is modelled as a normally distributed point load $\mathrm{P}$ with a mean value $\mu_{P}$ of $100 \mathrm{kN}$ and standard deviation $\sigma_{P}$ of $12 \mathrm{kN}$. Permanent loads are neglected in this example. It is assumed that the bending resistance 
(node "Resistance/Service limit" in Figure 1) is constant along the beam and is normally distributed with a mean value $\mu_{M_{R}}$ of $500 \mathrm{kNm}$ and standard deviation $\sigma_{M_{R}}$ of $40 \mathrm{kNm}$. The span (node "Span" in Figure 1 ) is $10 \mathrm{~m}$.

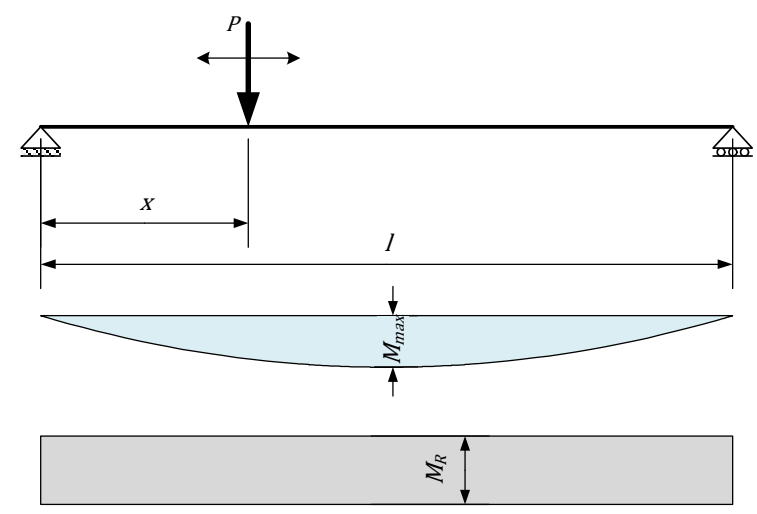

Figure 2. Load effect $M_{\max }$ and resistance $M_{R}$

The bridges are designed to withstand different combinations of actions and the corresponding structural checks can be used as a basis to define possible failure modes. For the example in Figure 2, three collapse mechanisms are chosen to be failure modes: FM1, FM2 and FM3 as shown in Figure 3. However, other failure modes can be selected based on owner's preferences. For instance, the extent of spalling or crack width can be chosen to be failure criteria.

If the reliability for FM1 is significantly lower than in $\mathrm{FM} 2$ and $\mathrm{FM} 3$, the contribution of the latter two can be neglected. Since the load effect and resistance are normally distributed, the reliability index, which means also the probability of failure for loading as in Figure 2 can be computed as follows:

$$
\begin{aligned}
\beta & =\frac{\mu_{M_{R}}-\frac{\mu_{P} \cdot l}{4}}{\sqrt{\sigma_{M_{R}}^{2}+\left(\frac{\sigma_{P} \cdot l}{4}\right)^{2}}} \\
& =\frac{500-250}{\sqrt{40^{2}+\left(\frac{120}{4}\right)^{2}}} \\
= & \frac{250}{40 \cdot \sqrt{\frac{25}{16}}}=\frac{1000}{200}=5 \Rightarrow \\
P_{f} & =2.87 \cdot 10^{-7}
\end{aligned}
$$

The reliability of undamaged bridge meets the criteria according to [1].

\subsection{Inspections and impact of damages}

An important constraint imposed to the methodology presented here is related to inspection procedure, which shouldn't change significantly. This constraint couldn't be completely satisfied as there is some additional information that is indispensable, if the effect of deterioration and damages is to be appropriately considered in assessment of bridges:

- Based on the design documentation, relevant failure modes need to be defined. These failure modes correspond to the critical load situations used in design and

- for each failure mode, vulnerable zones (as in [4] and [5]) are to be defined, in which damages have the largest impact on safety and serviceability.

VZ 1: High Saging Moment
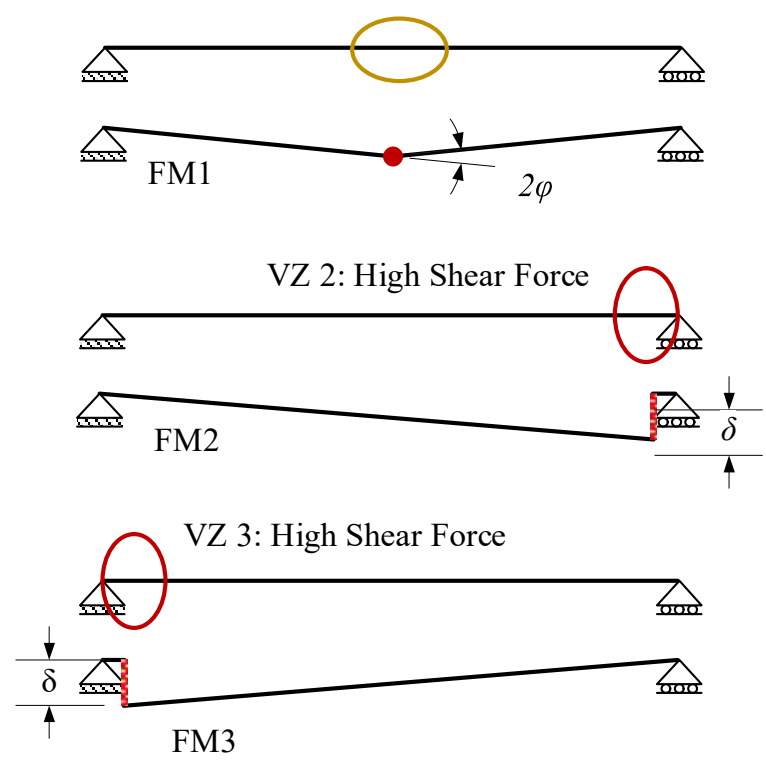

Figure 3. Vulnerable zones and failure modes

The definition of failure modes is already discussed in previous chapter. The vulnerable zones are closely linked to failure modes and experienced inspectors know intuitively where these zones are. Nevertheless, it is helpful if they can confirm themselves with the readily available information (see [6]). The damages outside vulnerable zones can also trigger failures, but for them to occur the extent of damage needs to be significantly larger than 
in the vulnerable zones. If this seems likely, one needs to define an additional failure mode that can be triggered by the observed damages.

For a simply supported beam in Figure 2 the vulnerable zones and corresponding failure modes are illustrated in Figure 3.

The definition of failure modes and vulnerable zones is mostly one-time activity and the modification are needed in case of significant changes in loading and/or resistance/service limits occurs.

The estimation of the impact of inspection (mostly visual) findings on reliability is up to now not seriously considered as a viable option. Visual inspections are considered to be subjective and uncertain allowing only qualitative outcome such as condition rating. Although it is undeniable that observations made during visual inspection are often fuzzy, they can be useful if their inherent uncertainty is properly modelled. In [7], a subset of observations collected in a survey are identified to have an impact on reliability. The corresponding uncertainties both regarding the inspection process and an impact on reliability are however not addressed, which remains to be the topic of a future research. In addition, there is also useful data that is simply not collected.

For instance, if merely "Corroded reinforcement" on a certain bridge element is reported, this means that the reinforcement corrosion can be anywhere on its elements i.e. its location needs to be uniformly distributed. Likewise, a spalling area and a section loss can be also modelled with slightly informative or non-informative distributions. If, however additional information is available such as that the reinforcement corrosion is located in vulnerable zone, the uncertainty with regard to its influence on reliability can be significantly reduced. The quantitative information on spalling area and section loss can further reduce uncertainty.

To consider the effect of inspection findings the Bayesian network in Figure 1 can be modified as shown in Figure 4.

In this example it is assumed that the visual inspection revealed a spalling area with the reinforcement corrosion (node "Corroded reinforcement" in Figure 4) with a section loss of $10 \%$. This is a typical entry as shown in the survey performed by the
WG1 of the COST Action 1406 (see [7]). The location of the defect is not known and there is inherent uncertainty with regard to this section loss. Based on experience or merely by engineering judgement the likelihood of section loss can be assumed as in the second row of Table 1.

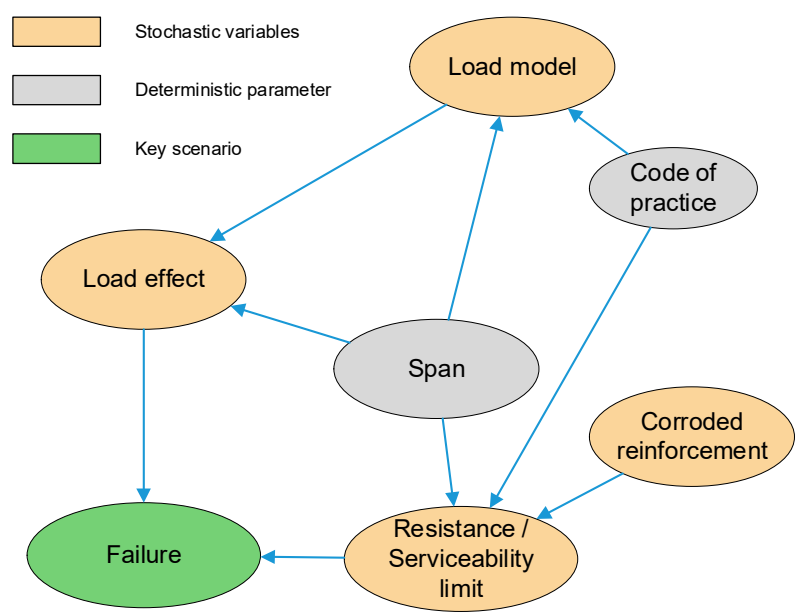

Figure 4. Reliability evaluation of damaged bridge

Table 1. Likelihood of indicating specific section loss

\begin{tabular}{c|ccccc}
\hline Section loss & $\mathbf{0 \%}$ & $\mathbf{5 \%}$ & $\mathbf{1 0 \%}$ & $\mathbf{1 5 \%}$ & $\mathbf{2 0 \%}$ \\
\hline $\begin{array}{c}\text { Probability } \\
\text { informative }\end{array}$ & $0 \%$ & $60 \%$ & $20 \%$ & $10 \%$ & $10 \%$ \\
\hline $\begin{array}{c}\text { Probability } \\
\text { non-informa- } \\
\text { tive }\end{array}$ & $20 \%$ & $20 \%$ & $20 \%$ & $20 \%$ & $20 \%$ \\
\hline
\end{tabular}

The third row expresses even less informative probability distribution in which all listed section losses are equally likely.

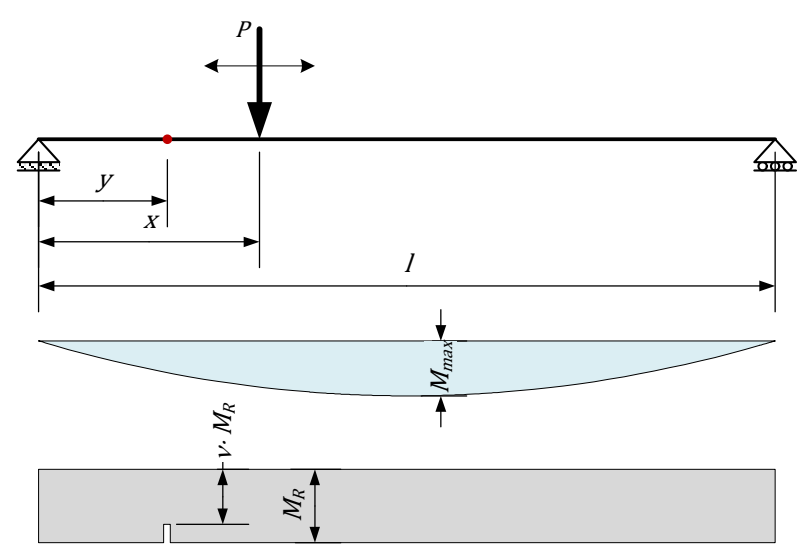

Figure 5. Resistance of damaged structure 
In the first step one needs to evaluate the safety index for the deterministic section loss with no information on its location. This means the safety index has to be computed for every possible location of a corroded reinforcement. The location of the corroded reinforcement is the unknown distance $y$, as in Figure 5.

The safety index cannot be larger than the one of the undamaged structure. It can be however lower if the section loss is in the area of high bending moments. This reasoning result in following expression for safety index.

$$
\begin{aligned}
& \beta\left(\frac{x}{l}\right)=\operatorname{Min}\left[\frac{v \cdot \mu_{M_{R}}-\mu_{P} \cdot\left(x-\frac{x^{2}}{l}\right)}{\sqrt{\sigma_{M_{R}}^{2}+\left(\sigma_{P} \cdot\left(x-\frac{x^{2}}{l}\right)\right)^{2}}}, 5\right] \\
& P_{f}=\Phi(-\beta)
\end{aligned}
$$

where $\Phi$ cumulative normal distribution with the mean value of 0 and standard distribution of 1.0. The safety index can be plotted as a function of location of corroded reinforcement. It can be seen in that for certain locations, the section loss has no influence on safety index. In the middle part, there is however a clear reduction of safety index indicating a vulnerable zone.

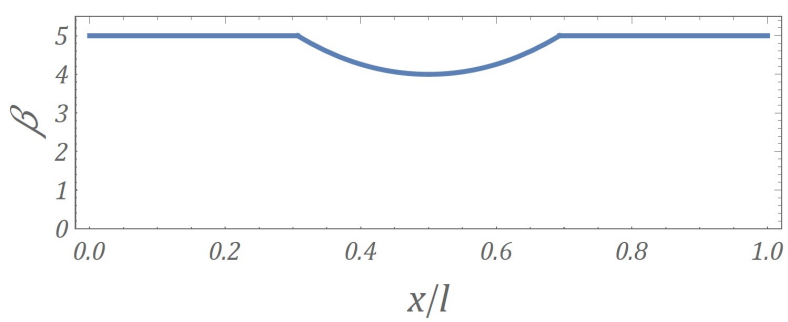

Figure 6. Reliability index in the function of damage location

Assuming that there is an equal probability of corroded reinforcement being anywhere on the beam one has to integrate the probability of failure for all locations as in following expression:

$$
P_{f}=\frac{1}{l} \int_{0}^{l} \Phi\left(-\beta\left(\frac{x}{l}\right)\right) \cdot d x
$$

In the second step this expression can be further refined by considering the uncertainty regarding section loss:

$$
P_{f}=\sum_{1}^{5} \vartheta_{i} \frac{1}{l} \int_{0}^{l} \Phi\left(-\beta\left(\frac{x}{l}\right)\right) \cdot d x
$$

where $\vartheta_{i}$ represents the likelihood values of the discrete distribution from Table 1. For the first row in the Table 1 the resulting probability of failure and corresponding safety index is as follows:

$$
P_{f}=3.15 \cdot 10^{-5} \Rightarrow \beta=4.0
$$

This means that the damaged structure doesn't meet the requirements of [1]. The probability of failure increased by the factor of 100 compared to the undamaged structure.

For the non-informative probability distribution of section loss presented in the third row of the Table 1 the probability of failure is as follows

$$
P_{f}=6.11 \cdot 10^{-5} \Rightarrow \beta=3.84
$$

The probability of failure doubled compared to the distribution of section loss assumed in the row 2 of Table 1.

\section{Conclusion}

In this paper, an approach is proposed to include results of visual inspections, which are often fuzzy, into assessment of reliability. It makes extensive use of information from design phase, which needs to be merely updated based upon the results of visual inspections. The approach closes a gap characteristic for todays practice, in which different performance indicators are used during the service life of a bridge.

The proposed approach uses Bayesian networks and to this end the Bayesian networks have to be adapted to accommodate relevant types of observations that are common in different countries. These observations are catalogue in common BMSs and a comprehensive list can be found in [7]. The impact of these observation to resistance/service limits is here crudely assumed and need to be further investigated. The same applies to uncertainty of observations. The likelihoods as in Table 1 need 
to be refined by investigating inspection practice in more detail.

Finally, it should be noted that the visual appearance of a bridge is not addressed in this paper, although it may play important role in decision-making process. Spalling concrete, dripping joints and corrosion traces are not very appealing and the owner or operator is inclined to remedy them in order to protect its reputation. The commonly used condition rating is often strongly influenced by visual appearance and indeed it can be used to evaluate it. A decent visual appearance can therefore be regarded as a performance goal as well. It is up to an owner or operator and the social environment to set up criteria for the decent visual appearance.

\section{References}

[1] CEN EN 1990, "Basis of structural design,» European Committee for Standardisation, Brussels, 2002.

[2] SIA, "SIA 269 - Maintenance of existing structures," Swiss Engineers and Architects Association, Zurich, 2011.

[3] R. Hajdin, M. Kušar, S. Mašović, P. Linneberg, J. Amado und N. Tanasić, "COST Action TU1406 Quality Specifications for Roadway Bridges (BridgeSpec),Technical report of the Working group 3: Establishment of a Quality Control Plan," 2018.

[4] P. Linneberg, S. Mašović und R. Hajdin, "Quality Control Plans for Girder and Frame Bridges," IABSE Symposium, Vancouver, 2017.

[5] NYSDOT, Concrete Details Vulnerability Manual, Albany, NY: New York State Departement of Transportation, 1997.

[6] R. Hajdin, "Visual inspections and key performance indicators - Bridging the gap," Transportation Research Circular, 11th International Bridge and Structures Management Conference, Nr. E-C224, October 2017a.
[7] A. Srauss und A. Mandić Ivanković, «COST Action TU1406 Quality Specifications for Roadway Bridges (BridgeSpec), Technical report of the Working group 1: Performance indicators of Cost Action TU1406, „ 2016. 